\title{
Adenocarcinoma of the bladder: case report
}

\begin{abstract}
Primary Adenocarcinoma of Bladder is an extremely rare and very aggressive form of cancer that forms about $1 \%$ of bladder cancer. It is suggested that it arises from intestinal metaplasia of the urothelium. ${ }^{1}$ It has a very aggressive course and a very poor response to the chemotherapy and radiotherapy. The curative therapy remains surgery if the cancer is confined to the bladder with no metastasis exist. ${ }^{2}$ We are presenting a case of an $83 \mathrm{yr}$ old male who was evaluated for gross hematuria and underwent TURBT which showed an adenocarcinoma of the bladder. No evidence of metastasis was found.
\end{abstract}

Keywords: male predominance, bladder irritation symptoms, endometrial and cervical, bladder source, extensive necrosis
Volume II Issue I - 2020

\author{
Chintav Shah,' Jay Patolia,' Simeon \\ Jaggernauth,' ${ }^{2}$ Nirav Nagarsheth' \\ 'Department of Internal Medicine, University of Arkansas for \\ Medical Sciences, USA \\ 2Landmark Cancer Center, Arkansas, USA
}

Correspondence: Chintav Shah, Department of Internal

Medicine, University of Arkansas for Medical Sciences,

Fayetteville, Arkansas, USA,

Email Chintavshah65@gmail.com, chshah@uams.edu

\section{Background}

Primary adenocarcinoma of the bladder is derived from the urothelium of the bladder. Patient present in the sixth to seventh decade of life with male predominance. ${ }^{2}$ The most common symptom on presentation is hematuria but they can also present as bladder irritation symptoms. ${ }^{2}$ Adult vesicle exostrophy and persistent Urachal remnants are also a risk factor for adenocarcinoma. ${ }^{3}$ The diagnosis of primary adenocarcinoma can only be made once the secondary causes has been ruled which mainly involves colorectal, endometrial and cervical. $^{2}$

\section{Case report}

This was an $83 \mathrm{yr}$ old male with $\mathrm{PMH}$ of an adenocarcinoma of the prostate treated with radiation in 1989. Patient states that he had 39 doses of radiation followed by seeds implantation several years later. He had surveillance with the PSA that remained low. He developed gross hematuria which was his presenting complaint. He underwent a TURBT in 2018 with pathology demonstrating evidence of an invasive adenocarcinoma that was moderately differentiated in a background of extensive necrosis. The cells stained positive for CDX2, CK20 and negative PSA. The primary sites were considered to be from colorectal or bladder source.

A PET scan was performed demonstrating several scattered sub centimeter and mildly avid mediastinal and bilateral hilar lymph nodes with SUV of 2.2. Intense activity in the cauterised urinary bladder is seen with an ill-defined mass in the cavity measuring $2.3 \times 2.5 \mathrm{~cm}$ with SUV of 65.0. Normal activity was identified throughout the skeleton.

Based on these findings, it was determined he had adenocarcinoma of the bladder arising possibly from urachus. Since the tumor was confined to the bladder with no adjacent invasion, the patient was deemed appropriate for a radical cystaprostectomy at a tertiary care center. $^{4}$

\section{Discussion}

Cases of primary adenocarcinoma of the bladder need to be approached with caution. Necessary investigations should be performed with imaging and appropriate screening as bladder adenocarcinomas are extremely rare. As in this case, despite all the imaging workup being negative, it was found to be a primary adenocarcinoma.

The urachus is the remnant of channel between the bladder and umbilicus where urine initially drains in the fetus during the first trimester of pregnancy. ${ }^{5}$ This channel seals off at the 12th week of gestation and all remains is a fibrous cord between the bladder and umbilicus called median umbilical ligament. ${ }^{5}$ It is the most reported tumor with exostrophic bladder (one of the urachal pathology) or after enterocystoplasty. ${ }^{6}$

The staging of adenocarcinoma is same as the urothelial carcinoma. ${ }^{1}$ Based on the staging, the treatment involves chemotherapy with 5-FU (the data on this is very limited with reported survival of 11 months), ${ }^{6}$ radiation therapy or surgery. Prognosis is poor with a $5 \mathrm{yr}$ survival rate of $18-47 \% .{ }^{1}$ Many cases presented with a higher stage including up to $40 \%$ with metastasis at the time of diagnosis. ${ }^{1}$ However if the initial tumor is confined within the bladder and is not involving the surrounding structures as in the above patient who was diagnosed early due to his symptoms, surgical resection of the bladder would present a good prognosis; however, the $5 \mathrm{yr}$ survival rate is still remains low. ${ }^{4}$

There has been no standard chemotherapy for the primary adenocarcinoma of the bladder. ${ }^{4}$ However it is suggested that $5 \mathrm{FU}$ based chemotherapy regimens can be utilized. A $5 \mathrm{FU}$ based regimen was recently replaced by S-1 which is novel fluoropyramidine derivate that consists of 5-chloro-2, 4-dyhydropyramidine (CDHP) and potassium oxonate. ${ }^{4}$

\section{Acknowledgments}

None.

\section{Conflicts of interest}

The authors declare that there is no conflict of interests regarding the publication of this paper.

\section{References}

1. American Urological Association

2. Dadhania V, Czerniak B, Guo CC. Adenocarcinoma of the urinary bladder. Am J Clin Exp Urol. 2015;3(2):51-63. 
3. Ball MW, Nathan R, Gerayli F. Long-term response after surgery and adjuvant chemoradiation for $\mathrm{t} 4$ mucinous adenocarcinoma of the bladder: a case report and review of the literature. Clin Genitourin Cancer. 2016;14(2):e225-e227.

4. $\mathrm{Xu} \mathrm{W}, \mathrm{Yu} \mathrm{B}, \mathrm{Xu} \mathrm{T}$, et al. Chemotherapy for primary adenocarcinoma of the urinary bladder: case report. Adv Pharmacoepidemiol Drug Saf. $2015 ; 4: 180$
5. https://urology.ucsf.edu/patient-care/children/urachal-abnormalities

6. el-Mekresh MM, el-Baz MA, Abol-Enein H, et al. Primary adenocarcinoma of the urinary bladder: a report of 185 cases. Br J Urol. 1998;82(2):206-212. 